MISS CANDELA PAESANI (Orcid ID : 0000-0002-6565-9266)

Article type : Original Article

\title{
Arabinoxylan from Argentinian whole wheat flour promote the growth of Lactobacillus reuteri and Bifidobacterium breve
}

\section{Arabinoxylans of Wheat with Potential Prebiotic Effect}

Candela Paesani $^{a, b, c}$, Emiliano Salvucci ${ }^{a, b, c}$, Malena Moiraghi ${ }^{a, c}$, Liliana Fernandez Canigia d, Gabriela T Pérez ${ }^{\mathrm{a}, \mathrm{c} * 1}$

a Institute of Food Science and Technology of Córdoba, (ICYTAC-CONICET-UNC), Córdoba, Argentina

${ }^{\mathrm{b}}$ Laboratory of Microbiology and Biotechnology, Faculty of Exact, Physics and Natural Science, National University of Córdoba, (FCEFYN-UNC)

${ }^{c}$ Faculty of Agronomy, National University of Córdoba, (FCA-UNC)

${ }^{\mathrm{d}}$ Microbiology Section, Central Laboratory, German Hospital, Buenos Aires, Argentina.

\section{SIGNIFICANCE AND IMPACT OF THE STUDY}

The present work demostrates that $\mathrm{AX}$ extracts from Argentinian soft and hard wheat promote efficiently the growth of probiotic strains L. reuteri ATCC23272 and B. breve 286, validated with three different parameters that consider the growth of representative strains of

$1{ }^{*}$ Corresponding author: Prof. Gabriela Pérez, Postal Address: Av Valparaíso s/n, Ciudad Universitaria, 5000-Córdoba, Argentina Tel: +54 3514334116 int 255

email: gaperez@agro.unc.edu.ar

This article has been accepted for publication and undergone full peer review but has not been through the copyediting, typesetting, pagination and proofreading process, which may lead to differences between this version and the Version of Record. Please cite this article as doi: 10.1111/lam.13097

This article is protected by copyright. All rights reserved. 
Bacteria genera found in the gut. The evaluation of $A X$ extracts as a food supplement in a murine model could confirm their ability to modulate the microbiome. Novel food prototypes including $A X$ and probiotics could relieve local symptoms and may act as psychobiotics with a beneficial effect on microbiome-brain axis.

\section{ABSTRACT}

Arabinoxylans are part of dietary fiber and have received attention given their emergent prebiotic character. Four arabinoxylans extracts were obtained from Argentinian soft and hard wheat. In vitro assays were performed to describe the extent to which the extracts from whole wheat flour support selective growth of Bifidobacterium breve and probiotic Lactobacillus reuteri ATCC23272 in a defined media. The prebiotic effect was evaluated by three quantitative scores: relative growth, prebiotic activity score and prebiotic index. For prebiotic index equation the growth of Bacteroides and Clostridium strains was compared to that of bifidobacteria and lactic acid bacteria. All the arabinoxylans extracts supported the growth of Lactobacillus and Bifidobacterium, reaching higher prebiotic activity score values than inulin (0.37 and 0.36 for Lactobacillus and Bifidobacterium, respectively). AX2 from soft wheat and AX4 from hard showed similar prebiotic index value to commercial inulin (2.64, 2.52 and 2.22, respectively), and AX3 extract presented higher prebiotic index value (4.09) than the positive control and other prebiotic index reported for arabinoxylans. These extracts could be used as prebiotic, synbiotic compositions or novel food prototypes to treat dysbiosis associated with many diseases.

Key words: dietary fiber, arabinoxylans, prebiotic index, Bifidobacterium, Lactobacillus

\section{INTRODUCTION}

Arabinoxylans $(\mathrm{AX})$ are the main non-starch polysaccharides in cereal cell walls and are part of dietary fiber. AX are classified according to their solubility in water extractable (WE-AX) and water unextractable (WU-AX). AX are formed by xylose chains (Xyl) bonded by $\beta$ 1-4 
bond, substituted by arabinose units (Ara) bonded by $1 \alpha-2$ and $1 \alpha-3$ bonds along the xylose chain.

The development of prebiotic therapies has become an interesting strategy for the amelioration of dysbiosis associated with several diseases. The modulation of beneficial bacteria can result in a regulatory effect not only locally but also at the immunological or neuroendocrine levels (Salvucci, 2016; Davis, 2016; Sarkar et al., 2016). Moreover, it impacts on the central nervous system, modifying levels of neurotransmitters and neurotrophins (Zhou and Foster, 2015; Bindemann and Ali, 2018).

The prebiotic effect on the intestine can be evaluated by the proliferation of lactobacilli and bifidobacteria. The bifidogenic and growth-promoting effect of probiotic strains is desirable in the search of synbiotic supplements and novel food prototypes. For instance, the development of a formula milk for infants who cannot be breastfed or a prebiotic supplement to treat adult dysbiosis where the bifidogenic effect can exert a metabolic change and alleviate symptoms are novel applications of prebiotics and probiotics. In addition, the effect of prebiotic and probiotics on microbiome-brain axis opens the possibility of developing "psychobiotics" to confer mental health benefits (Sarkar et al., 2016).

The aim of this work was to determine the in vitro prebiotic activity of WE-AX extracts obtained from whole wheat flours. The relative growth, prebiotic activity score and prebiotic index was calculated using Lactobacillus reuteri ATCC23272, Bifidobacterium breve 286, Bacteroides fragilis 6292 and Clostrodium perfringens 4168 strains. These indexes are valuable tools to estimate the ability to modulate microbiome and to define forthcoming in vivo assays. The correlation between these parameters and WE-AX sugar composition was also assessed.

\section{RESULTS AND DISCUSSION}

The WE-AX content, sugar composition and the ratio Ara/Xyl of each extract are shown in Table 1. The content of WE-AX varied from 39 to $58 \%$ and all the extracts exhibited greater amounts of xylose (Xyl) than arabinose (Ara) residues. Significant differences between AX2 
and the other samples were observed. Extracts from AX2 showed the highest Ara/Xyl ratio. Saulnier et al. (2007) also reported values of xylose similar as the values found in this work

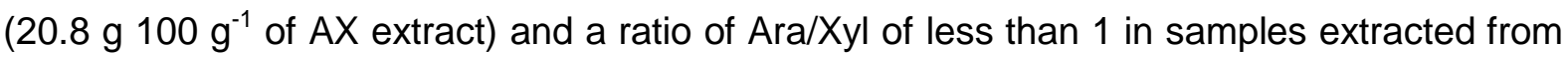
wheat bran, refined and whole wheat flour. Zhou et al. (2010) and Barron et al. (2007) also found similar values of Ara/Xyl. The AX4 showed a high glucose content, unlike the other extracts.

Figure 1 shows the relative growth ratio $(R G)$ of probiotic L. reuteri ATCC23272 after a $24 \mathrm{~h}$ incubation period in a semidefined broth that contained AX extracts and commercial inulin $R G$ as carbon source, respectively. The growth of the probiotic strain in all cases was higher with $A X$ than the growth with glucose. RG was higher than 1 and reached 1.2 with AX3.

Bifidobacterium breve 286 grew efficiently in this semidefined media plus AX. The values were higher than those obtained with commercial inulin. Moreover, the RG at $24 \mathrm{~h}$ for these AX extracts were higher than previous reports for inulin extracts (Rubel et al., 2014).

Differences in growth promotion between samples can result from structural differences. WE-AX extracts with lower Ara/Xyl ratio showed the highest Lactobacillus growth, however no significant correlations between Ara/Xyl ratio and $\mathrm{RG}$, prebiotic activity or prebiotic index were observed. Other characteristics such as WE-AX molecular size or degree of polymerization or substitution could be influencing the prebiotic capacity of extracts. These structural differences in $A X$ from wheat affect the ability of bacteria to degrade it. Fermentation by bifidobacteria, clostridia, bacteroides and lactobacilli differed with the oligosaccharide structure (Toole et al., 2011; van den Abeele, 2013; van Laere et al.,1997). This first parameter of prebiotic activity showed that with all of the AX extracts, RG of both probiotic bacteria, L. reuteri ATCC23272 and B. breve 286, were equal or greater than the positive control (Figure 1). These results show the ability of these probiotic bacteria to metabolize AX efficiently. It is known that Bifidobacterium can metabolize $A X$ and the genomic analysis revealed that this ability is strain-dependent. There are reports of extracellular enzymes involved in AX degradation (Rivière et al., 2014).

This article is protected by copyright. All rights reserved. 
The PAS was calculated for L. reuteri, B. breve, C. perfringens and B. fragilis (Table 2). PAS obtained with commercial inulin and the four $\mathrm{AX}$ extracts using Bifidobacterium and Lactobacillus strains are shown in Figure 2. Higher PAS indicate higher relative growth of the probiotic and/or lower relative growth of the enterobacteria. In any of these cases, the prebiotic is more selectively used in relation to glucose by the probiotic microorganism or enterobacteria shows a limited use of the prebiotic in relation to glucose (Rubel et al., 2014). The values reported were all equal or greater than inulin with $B$. breve 286 and $L$. reuteri ATCC23272, except for AX2 (Figure 2). AX1 exhibited similar values than inulin in both probiotic strains. AX3 and AX4 from hard wheat showed significant higher PAS than inulin for both strains. AX extracts from hard wheat showed higher PAS than inulin for L. reuteri and $B$. breve. These differences could be attributed to structural heterogeneity of $A X$. It is known that wheat varieties show diversity in $A X$ structure from low-substituted $A X$ to almost entirely of highly substituted $A X$ (Toole et al., 2011). It has an impact on the solubility of $A X$ and the ability of bacteria to degrade them (Riviére et al., 2014).

The PI takes into account the growth not only of both probiotic strains but also of $B$. fragilis and $C$. pergringens. This allows the quantification of the prebiotic effect, given a more precise index than evaluations that are solely qualitative and based on the growth of key bacterial groups during fermentation (Palframan et al., 2003). It is a more accurate index since it considers not only the bifidogenic effect but also the growth of non-probiotic strains.

The PI for AX2 and AX4 showed a similar value to commercial inulin (2.22) (Table 2). AX3 exhibited a higher value than inulin and other PI reported for AX (Vardakou et al., 2008). The highest PAS for Lactobacillus and the highest PI were obtained with

hard wheat-derived AX3. AX1 showed the lowest PI since it supported the highest growth of B. fragilis. Vardakou et al., 2008 obtained a PI of 1.15 and 2.42 for AX treated and untreated with xylanase, respectively (Figure 3). In our study Bifidobacterium was able to ferment AX extracts as was observed previously (van den Abbeele et al., 2011a, van den Abbeele, van de Wiele and Possemiers, 2011b).

This article is protected by copyright. All rights reserved. 
These PI values for $\mathrm{AX} 2, \mathrm{AX} 3$ and $\mathrm{AX} 4$ are higher than those reported for commercial fructooligosaccharides and galactooligosacharides (Palframan et al., 2003). AX1 from soft wheat flours with lower concentration of pentosans exhibited the lowest PI (Figure 3).

The success of $A X$ extracts in producing a growth increase of $B$. breve is similar or better than the effect observed with inulin (Grootaert et al., 2009; Rubel et al., 2014). These results confirm the efficacy of AX and support its effect as a prebiotic (Grootaert et al., 2009; van den Abbeele et al., 2011a). The combination of AX with Bifidobacterium strains is a possibility to develop innovative milk formulas for infants that cannot be breastfed.

L. reuteri ATCC23272 produces reuterin, an antimicrobial that protects from pathogens, and histamine with anti-inflammatory effect (Cadieux et al., 2008). Moreover, it has shown the ability to reduce the incidence of necrotizing enterocolitis by $50 \%$ in a well-defined animal model (Navarro et al., 2017). The AX extracts evaluated in this work demonstrate that they can support the growth of this probiotic. These results suggest the possibility to combine $A X$ with the probiotic strain in a synbiotic formula to enhance their effects at the gastrointestinal level.

The incorporation of total bacterial numbers in the PI becomes important because it is impossible to routinely enumerate all the bacterial groups resident in the human colon. It may well be the case that a large increase in bifidobacteria is observed in a prebiotic study together with an increase in unrecognized and less beneficial bacterial groups that are not enumerated. Bacterial groups of interest are therefore identified according to whether or not strain growth is beneficial.

Lactobacilli, bacteroides and clostridia non-pathogen strains are specialized in the degradation of complex carbohydrates. They play an important role in carbohydrate metabolism because of their strain-dependent ability to produce a wide range of depolymerizing enzymes (Grootaert et al., 2007). When fermenting dietary fibers in colon, bifidobacteria produce SCFA that decrease the $\mathrm{pH}$ of the intestine and negatively affect the growth of potentially pathogenic bacteria ( $\mathrm{Li}$ et al., 2015). They also have the ability to produce vitamins and exert beneficial effects like immune stimulation, reduction of intestinal 
transit time, decrease of serum cholesterol levels, among others (van der Meulen et al., 2016).

B. fragilis has endoxylanase activity and arabinosidases that allow to degrade AX. The high values of PAS obtained for this strain can be explained by this ability. The increase in Bacteroides would not be an undesirable result since this group with its endoxylanase activity produces oligosaccharides that are metabolized by Bifidobacterium strains. Furthermore, the increase in Bacteroides was associated with the production of beneficial SCFA (De Filippo et al., 2010). The main effect of dietary fiber such as AX on microbiome is associated with anti-obesity effect due to the synergistic effect of promoting Bacteroides and Bifidobacterium growth. The production of SCFA and their effect on gut barrier maintenance reduces the risk of endotoxemia and inflammation. L. reuteri ATCC23272 has shown antiinflammatory effects. The combined effect of $A X$ and anti-inflammatory probiotics opens the possibility to develop novel food prototypes with prebiotic applications.

In conclusion, AX extracts obtained from different varieties of Argentinian wheat showed a potential prebiotic effect validated with three different parameters that consider the growth of representative strains of Bacteria genera found in the gut. AX obtained from hard wheat varieties has a prebiotic effect and shows better values than commercial inulin. We showed that $\mathrm{AX}$ extracts promote efficiently the growth of probiotic strains L. reuteri ATCC23272 and B. breve 286 .

Forthcoming evaluation of $A X$ extracts as a food supplement in a murine model could confirm their ability to modulate the microbiome. This will allow to evaluate the extent to which the bifidogenic capacity and $L$. reuteri growth-promoting properties can alleviate symptoms of different diseases related to microbiome dysbiosis. Novel food prototypes including $A X$ and probiotics could relieve local symptoms and may act as psychobiotics with a beneficial effect on microbiome-brain axis.

This article is protected by copyright. All rights reserved. 


\section{MATERIALS AND METHODS}

\section{Raw material}

Nine hard wheat cultivars: Cronox, Klein Yarará, Klein Guerrero, Aniversario 75, Biointa 3004, Baguette 11, ACA 315, ACA 320, LE 2330 and 12 experimental lines of soft wheat (PM 647, 650, 663, 673, 679, 681, 682, 686, 687, 690, 691 and 692) (INTA Marcos Juarez, Córdoba, Argentina) were selected for WE-AX extraction. The cultivars of hard wheat bread were those most widely cultivated in Argentina during the last three seasons. Experimental lines of soft wheat were selected by cookie quality (Moiraghi et al., 2011). The grains were conditioned to a moisture content of $15 \%$ and ground in a roller mill without sieves. Wholemeal flours were stored at $-20^{\circ} \mathrm{C}$ to avoid lipid degradation.

\section{Extraction of water-soluble arabinoxylans (WE-AX)}

Four groups were formed according to the content of soluble pentosanes measured following Orcinol - $\mathrm{HCl}$ method (Hashimoto et al., 1987) in the flours from different genotypes. AX 1: soft wheats with a WE-AX concentration of less than $0.7 \%$ (w/w) (PM 663, PM 681, PM 673); AX 2: soft wheats with a WE-AX concentration greater than $0.7 \%(w / w)$ percentage in dry weight) (PM 682, PM 650); AX 3: hard wheats with a WE-AX concentration of less than $0.7 \%(w / w)$ (Cronox, ACA 320, Baguette), and AX 4: hard wheats with a WE-AX concentration greater than $0.7 \%$ (w/w) (Klein Guerrero, Klein Yarará).

The water-soluble AX extraction technique was set up following the methodology of Buksa et al. (2010). The yields obtained (between 1.09 and $1.89 \%$ ) correspond roughly to the content of $A X$ in the samples.

\section{Characterization of arabinoxylan extracts}

$25 \mathrm{mg}$ of WE-AX were hydrolysed with $2 \mathrm{~mL}$ of $2 \mathrm{~mol} \mathrm{I}^{-1}$ TFA for two $\mathrm{h}$ at $100{ }^{\circ} \mathrm{C}$. The hydrolysate was centrifuged at $2000 \mathrm{~g}$ for five $\min .3 .4 \mathrm{~mL}$ of distilled water was added to 1.6 $\mathrm{mL}$ of the supernatant and neutralized with $\mathrm{Na}_{2} \mathrm{CO}_{3}$. The obtained solution was filtered with a pore size of $22 \mu \mathrm{m}$, and the samples were injected into the HPLC. A column of Supelco Ca, 
at $80^{\circ} \mathrm{C}$, with water as the mobile phase and a flow of $0.5 \mathrm{~mL} \mathrm{~min}{ }^{-1}$ with a RID detector was used. The samples were processed in duplicate. A calibration curve was made for arabinose, xylose and glucose with SUPELCO standards (Monosaccharide Kit 47267) treated in the same way as the samples.

\section{Strains and media}

Lactobacillus reuteri ATCC23272, Bifidobacterium breve 286 (ICYTAC), Bacteroides fragilis 6292 and Clostrodium perfringens 4168 (Microbiology Section, Hospital Alemán) were grown in MRS media. Escherichia coli ATCC25922 were grown in Brain Heart Infusion (BHI) broth. For prebiotic effect assays, a semi-defined medium was used based on Vernazza et al. (2005) and Zhang et al. (2013): peptone $0.15 \%(w / v)$; yeast extract $0.2 \%(w / v) ; \mathrm{K}_{2} \mathrm{HPO}_{4}$ $0.004 \%(w / v) ; \mathrm{KH}_{2} \mathrm{PO}_{4} 0.004 \%$ (w/v); $\mathrm{MgSO}_{4} .7 \mathrm{H}_{2} \mathrm{O} 0.004 \%$ (w/v); $\mathrm{NaHCO}_{3} 0.02 \%$ (w/v); Tween $800.2 \%(v / v)$; bile salts $0.05 \%(w / v)$; $\mathrm{CaCl} 0.001 \%(\mathrm{w} / \mathrm{v})$.

\section{Prebiotic effect}

The test was carried out following the method of Huebner et al., (2007) with modifications. 1 $\%(w / v)$ glucose or $1 \%(w / v)$ of the AX extracts (Rivière et al., 2014; Rubel et al., 2014) was added to semidefinite broth. It was inoculated with $1 \%(\mathrm{v} / \mathrm{v})$ of an overnight culture of each of the strains. Cultures were incubated at $37^{\circ} \mathrm{C}$ under anaerobic conditions in the case of $B$. breve, C. perfringens and B. fragilis, and under aerobic conditions for L. reuteri and E. coli. At 0 and $24 \mathrm{~h}$ of incubation, $\mathrm{CFU} \mathrm{mL} \mathrm{mL}^{-1}$ counts of each of the inoculums were made using MRS agar in all cases, except for E. coli that were seeded on $\mathrm{BHI}$ agar. They were incubated for $24 \mathrm{~h}$ at $37^{\circ} \mathrm{C}$. Each assay was performed in duplicate and commercial inulin was included as a positive control.

This article is protected by copyright. All rights reserved. 
Relative growth (RG) and prebiotic activity score (PAS)

The RG was calculated by comparing the growth of the same strain incubated in the medium with glucose and in the one supplemented with the $\mathrm{AX}$, according to this equation: $R G=$ $(A X 24-A X 0) /(G 24-G 0)$. Where $A X 24$ and $A X 0=$ bacterial number after 24 and $0 \mathrm{~h}$ in the medium with $\mathrm{AX}$ and $\mathrm{G} 24$ and $\mathrm{G} 0=$ bacterial number after 24 and $0 \mathrm{~h}$ in glucose medium. The PAS was determined using the equation from Huebner et al., (2014) that is based on $R G$ of the probiotic strain relative to the $R G$ of the enteric bacteria $E$. coli. If the $A X$ has prebiotic activity, it will increase the growth of the probiotic bacteria, B. breve and L. reuteri ATCC23272, in relation to their growth in the same culture medium but supplemented only with glucose. That growth, in turn, must be greater than the enterobacteria strain, which is not fermenting the prebiotic compound.

\section{Prebiotic index $(P I)$}

The PI was calculated according to Palframan et al (2003). The equation was $\mathrm{Pl}=$ $($ Lact/Total $)-($ Bact/Total $)+($ Bif/Total $)-($ Clost/Total $)$, where Lact, Bact, Bif and Clost are CFU of each strain (L. reuteri ATCC23272, B. fragilis 6292, B. breve 286 and C. perfringens 4168, respectively) as a function of the total CFU. If a bacterial group shows a greater relative increase than does the total bacterial population, a $\mathrm{PI}>1$ is obtained if the relative increase is lower than the total bacterial increase $\mathrm{PI}<1$ is obtained.

\section{Statistical analysis}

Analysis of variance (ANOVA) was performed to determine statistical significance of the observed differences (Infostat software). Pearson correlation coefficient was calculated to determine the relationship between the sugar composition of the extracts and their prebiotic behaviour. 


\section{ACKNOWLEDGEMENTS}

The authors acknowledge Gabriela Diaz Cortez and Prof. Brenda Burgos for providing useful suggestions to improve the English in this manuscript. Authors would like to thanks CONICET and the Agencia Nacional de Promoción Científica y Tecnológica (Grant PICT 2015-0606) for their financial support.

\section{CONFLICT OF INTEREST}

No conflict of interest declared

\section{REFERENCES}

Barron, C., Surget, A. and Rouau, X. (2007) Relative amounts of tissues in mature wheat (Triticum aestivum L.) grain and their carbohydrate and phenolic acid composition. J Cereal Sci. 45, 88-96.

Bindemann, N. and Ali, S. (2018) The gut-brain connection: the developing science of the microbiome. Prog Neurol Psychiatry. 22, 4.

Buksa, K., Nowotna, A., Praznik, W., Gambuś, H., Ziobro, R. and Krawontka, J. (2010) The role of pentosans and starch in baking of wholemeal rye bread. Food Res Int. 43, 2045-2051.

Cadieux, P., Wind, A., Sommer, P., Schaefer, L., Crowley, K., Britton, R.A. and Reid, G. (2008) Evaluation of Reuterin Production in Urogenital Probiotic Lactobacillus reuteri RC-14. Appl Environ Microbiol. 74, 4645-4649.

Davis, C.D. (2016) The Gut Microbiome and Its Role in Obesity. Nutr Today. 51, 167-174.

De Filippo, C., Cavalieri, D., Di Paola, M., Ramazzotti, M., Poullet, J.B., Massart, S., Collini, S., Pieraccini, G. and Lionetti, P. (2010) Impact of diet in shaping gut microbiota revealed by a 
comparative study in children from Europe and rural Africa. Proc Natl Acad Sci U S A. 107, 14691-14696.

Grootaert, C., Delcour, J.A., Courtin, C.M., Broekaert, W.F., Verstraete, W. and van de Wiele, T. (2007) Microbial metabolism and prebiotic potency of arabinoxylan oligosaccharides in the human intestine. Trends Food Sci Technol. 18, 64-71.

Grootaert, C., van den Abbeele, P., Marzorati, M., Broekaert, W.F., Courtin, C.M., Delcour, J.A., Verstraete, W. and van de Wiele, T. (2009) Comparison of prebiotic effects of arabinoxylan oligosaccharides and inulin in a simulator of the human intestinal microbial ecosystem. FEMS Microbiol Ecol. 69, 231-242.

Hashimoto, S., Pomeranz, Y. and Shogren, M.D. (1987). Cereal pentosans: Their estimation and significance. I. Pentosans in wheat and milled wheat products. Cereal Chem. 64, 30-34.

Huebner, J., Wehling, R.L. and Hutkins, R.W. (2007) Functional activity of commercial prebiotics. Int Dairy J. 17, 770-775.

Li, Z., Summanen, P.H., Komoriya, T. and Finegold, S.M. (2015) In vitro study of the prebiotic xylooligosaccharide (XOS) on the growth of Bifidobacterium spp and Lactobacillus spp. Int J Food Sci Nutr. 66, 919-922.

Moiraghi, M., Vanzetti, L., Bainotti, C., Helguera, M., Leon, A. and Perez, G. (2011) Relationship Between Soft Wheat Flour Physicochemical Composition and Cookie-Making Performance. Cereal Chem. 88, 130-136.

Navarro, J.B., Mashburn-Warren, L., Bakaletz, L.O., Bailey, M.T. and Goodman, S.D. (2017) Enhanced Probiotic Potential of Lactobacillus reuteri When Delivered as a Biofilm on Dextranomer Microspheres That Contain Beneficial Cargo. Front Microbiol. 8, 489.

This article is protected by copyright. All rights reserved. 
Palframan, R., Gibson, G.R. and Rastall, R.A. (2003) Development of a quantitative tool for the comparison of the prebiotic effect of dietary oligosaccharides. Lett Appl Microbiol. 37, 281284.

Rivière, A., Moens, F., Selak, M., Maes, D., Weckx, S. and De Vuyst, L. (2014) The Ability of Bifidobacteria To Degrade Arabinoxylan Oligosaccharide Constituents and Derived Oligosaccharides Is Strain Dependent. Appl Environ Microbiol. 80, 204-217.

Rubel, I.A., Pérez, E.E., Genovese, D.B. and Manrique, G.D. (2014) In vitro prebiotic activity of inulinrich carbohydrates extracted from Jerusalem artichoke (Helianthus tuberosus L.) tubers at different storage times by Lactobacillus paracasei. Food Res Int. 62, 59-65.

Salvucci E. (2016) Microbiome, holobiont and the net of life. Crit Rev Microbiol 42(3):485-94.

Sarkar, A., Lehto, S.M., Harty, S., Dinan, T.G., Cryan, J.F. and Burnet, P.W.J. (2016) Psychobiotics and the Manipulation of Bacteria-Gut-Brain Signals. Trends Neurosci. 39, 763-781.

Saulnier, L., Sado, P.-E., Branlard, G., Charmet, G. and Guillon, F. (2007) Wheat arabinoxylans: Exploiting variation in amount and composition to develop enhanced varieties. J Cereal Sci, The Contribution of Cereals to a Healthy Diet. 46, 261-281.

Toole, GA, Le Gall, G, Colquhoun, IJ, Johnson, P, Bedo, Z, Saulnier, L, Shewry, PR, Mills, EN. (2011) Spectroscopic analysis of diversity of Arabinoxylan structures in endosperm cell walls of wheat cultivars (Triticum aestivum) in the HEALTHGRAIN diversity collection. J Agric Food Chem. 59(13), 7075-82.

van den Abbeele, P., Gérard, P., Rabot, S., Bruneau, A., El Aidy, S., Derrien, M., Kleerebezem, M., Zoetendal, E.G., Smidt, H., Verstraete, W., van de Wiele, T. and Possemiers, S. (2011a) Arabinoxylans and inulin differentially modulate the mucosal and luminal gut microbiota and mucin-degradation in humanized rats. Environ Microbiol. 13, 2667-2680.

This article is protected by copyright. All rights reserved. 
van den Abbeele, P., van de Wiele, T. and Possemiers, S. (2011b) Prebiotic effect and potential health benefit of arabinoxylans. Agro Food Ind Hi-Tech. 22, 9-12.

van den Abbeele, P, Venema, K, Van de Wiele, T, Verstraete, W, Possemiers, S. (2013) Different human gut models reveal the distinct fermentation patterns of Arabinoxylan versus inulin. $J$ Agric Food Chem. 61(41), 9819-27.

van der Meulen, T.A., Harmsen, H., Bootsma, H., Spijkervet, F., Kroese, F. and Vissink, A. (2016) The microbiome-systemic diseases connection. Oral Dis. 22, 719-734.

Van Laere K.M.J., Bosveld M., Schols H.A., Beldman G., Voragen A.G.J., 1997. Fermentative degradation of plant cell wall derived oligosaccharides by intestinal bacteria. Proceeding of the International Symposium "Non-Digestible Oligosaccharides: Healthy Food for the Colon?". Wageningen, The Netherlands, pp. 37-46

Vardakou, M., Palop, C.N., Christakopoulos, P., Faulds, C.B., Gasson, M.A. and Narbad, A. (2008) Evaluation of the prebiotic properties of wheat arabinoxylan fractions and induction of hydrolase activity in gut microflora. Int J Food Microbiol. 123, 166-170.

Vernazza, C.L., Gibson, G.R. and Rastall, R.A. (2005) In vitro fermentation of chitosan derivatives by mixed cultures of human faecal bacteria. Carbohydr Polym. 60, 539-545.

Zhang, N., Huang, X., Zeng, Y., Wu, X. and Peng, X. (2013) Study on prebiotic effectiveness of neutral garlic fructan in vitro. Food Sci Hum Wellness. 2, 119-123.

Zhou, L. and Foster, J.A. (2015) Psychobiotics and the gut-brain axis: in the pursuit of happiness. Neuropsychiatr Dis Treat. 11, 715-723.

This article is protected by copyright. All rights reserved. 
Zhou, S., Liu, X., Guo, Y., Wang, Q., Peng, D. and Cao, L. (2010) Comparison of the immunological activities of arabinoxylans from wheat bran with alkali and xylanase-aided extraction.

Carbohydr Polym. 81, 784-789.

This article is protected by copyright. All rights reserved. 
Table 1. The WE-AX content and the ratio Ara/Xyl

\begin{tabular}{cccccc}
\hline Extract & WE-AX \% & Xyl $\left(\mathrm{mg} \mathrm{g}^{-1}\right)$ & Ara $\left(\mathrm{mg} \mathrm{g}^{-1}\right)$ & Glu $\left(\mathrm{mg} \mathrm{g}^{-1}\right)$ & Ara/Xyl \\
\hline AX1 & $(39 \pm 2)^{\mathrm{a}}$ & $(227 \pm 8)^{\mathrm{a}}$ & $(107 \pm 2)^{\mathrm{a}}$ & $(1.6 \pm 0.1)^{\mathrm{a}}$ & $(0.47 \pm 0.04)^{\mathrm{a}}$ \\
AX2 & $(58 \pm 4)^{\mathrm{c}}$ & $(167 \pm 3)^{\mathrm{a}}$ & $(109 \pm 1)^{\mathrm{a}}$ & $(1.0 \pm 0.1)^{\mathrm{a}}$ & $(0.64 \pm 0.02)^{\mathrm{b}}$ \\
AX3 & $(45 \pm 3)^{\mathrm{b}}$ & $(283 \pm 2)^{\mathrm{b}}$ & $(115 \pm 2)^{\mathrm{a}}$ & $(44.7 \pm 0.5)^{\mathrm{b}}$ & $(0.41 \pm 0.01)^{\mathrm{a}}$ \\
AX4 & $(44 \pm 1)^{\mathrm{b}}$ & $(300 \pm 9)^{\mathrm{b}}$ & $(150 \pm 7)^{\mathrm{b}}$ & $(247 \pm 7)^{\mathrm{c}}$ & $(0.50 \pm 0.01)^{\mathrm{a}}$
\end{tabular}

Ara=Arabinosa, $\mathrm{Xyl}=\mathrm{Xylose}, \mathrm{Glu}=$ Glucose and Ara/Xyl= Arabinose/xylose ratio. Samples with a common letter in the same row are not significantly different $(p=0.05)$.

Table 2. The WE-AX Prebiotic Activity Score and Prebiotic Index

\section{Carbon source}

\begin{tabular}{|c|c|c|c|c|c|}
\hline Strain & Inulin & AX 1 & $A \times 2$ & AX 3 & AX 4 \\
\hline \multirow[t]{2}{*}{ Clostridium } & $(0.21 \pm 0.09)$ & $(0.15 \pm 0.01)$ & $(0.15 \pm 0.01$ & $(0.07 \pm 0.00)$ & $(0.15 \pm 0.01)$ \\
\hline & a & a & )$^{a}$ & a & a \\
\hline \multirow[t]{2}{*}{ Bacteroides } & $(0.41 \pm 0.02)$ & $(0.61 \pm 0.02)$ & $(0.39 \pm 0.03$ & $(0.52 \pm 0.03)$ & $(0.51 \pm 0.03)$ \\
\hline & $\mathrm{a}$ & b & )$^{a}$ & $a b$ & $\mathrm{ab}$ \\
\hline \multirow[t]{2}{*}{ Lactobacillus } & $(0.18 \pm 0.01)$ & $(0.17 \pm 0.01)$ & $(0.06 \pm 0.00$ & $(0.37 \pm 0.02)$ & $(0.27 \pm 0.02)$ \\
\hline & $\mathrm{ab}$ & $\mathrm{ab}$ & )$^{a}$ & c & $b c$ \\
\hline Bifidobacteriu & $(0.28 \pm 0.01)$ & $(0.23 \pm 0.02)$ & $(0.25 \pm 0.02$ & $(0.36 \pm 0.02)$ & $(0.33 \pm 0.01)$ \\
\hline$m$ & a & a & )$^{a}$ & $\mathrm{a}$ & a \\
\hline Prebiotic & $(2.61 \pm 0.02)$ & $(0.06 \pm 0.00)$ & $(2.64 \pm 0.03$ & $(4.09 \pm 0.03)$ & $(2.52 \pm 0.02)$ \\
\hline Index & $\mathrm{b}$ & a & )$^{\mathrm{b}}$ & c & b \\
\hline
\end{tabular}

Means values for the same bacterium with a common letter in the same row are not significantly different $(p=0.05)$. 
Figure 1. Relative growth ratio of L. reuteri ATCC23272 and B. breve 286

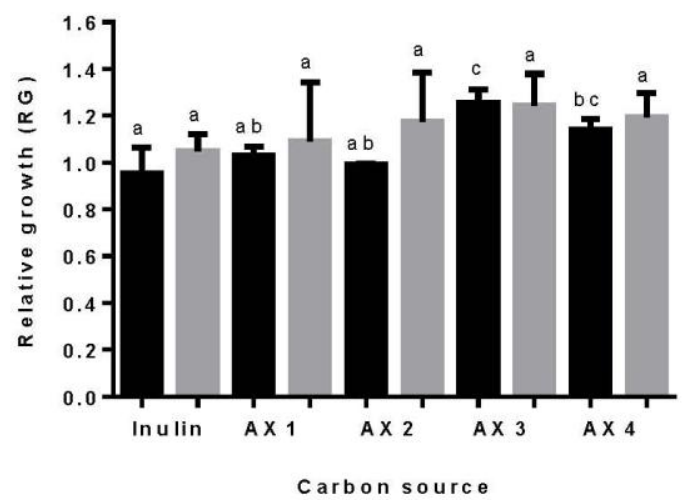

Relative growth ratio of $L$. reuteri ATCC23272 (black) and B. breve 286 (gray) with different $A X$-rich carbohydrate samples $(A X 1, A X 2, A X 3, A X 4)$ and commercial inulin. Means values with a common letter are not significantly different $(p=0.05)$.

This article is protected by copyright. All rights reserved. 
Figure 2. Prebiotic activity score (PAS) for B. breve 286 and L. reuteri ATCC23272

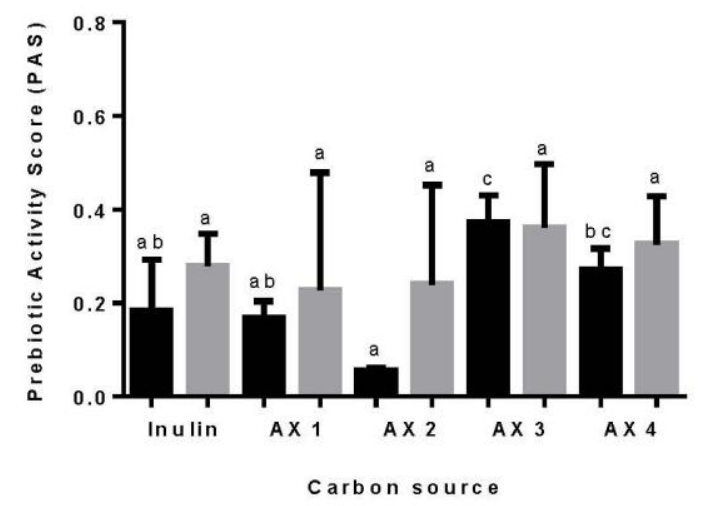

Prebiotic activity score (PAS) calculated for the different substrates for B. breve 286 (gray) and L. reuteri ATCC23272 (black). Means values for the same bacterium with a common letter are not significantly different $(p=0.05)$.

This article is protected by copyright. All rights reserved. 
Figure 3. The WE-AX Prebiotic Index scores

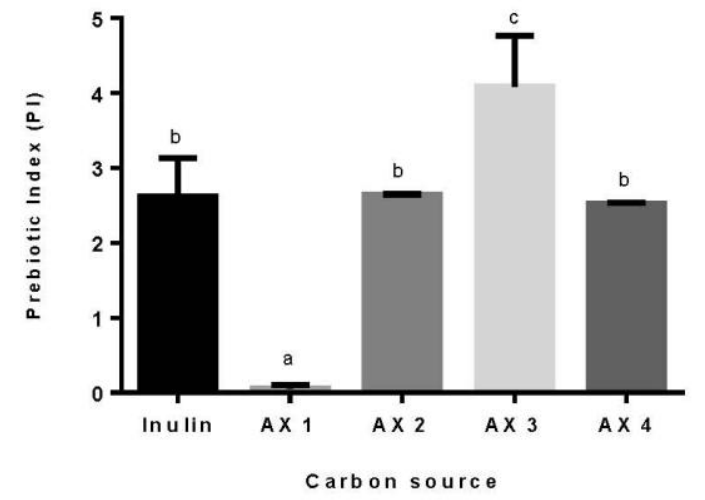

Prebiotic Index scores from batch culture fermentation of $1 \%$ test $A X$ samples and inulin.

Means values with different letters are significantly different.

This article is protected by copyright. All rights reserved. 